\title{
Amantadine for freezing of gait in patients with Parkinson's disease
}

\author{
Roneil Malkani, MD, MS ${ }^{1,{ }^{*}}$, Cindy Zadikoff, MD, MSc ${ }^{1}$, Onur Melen, MD ${ }^{1}$, Aleksandar \\ Videnovic, MD, MSc ${ }^{1}$, Emily Borushko, MPH ${ }^{1}$, and Tanya Simuni, MD ${ }^{1}$ \\ ${ }^{1}$ Department of Neurology, Northwestern University, Chicago, IL, USA
}

\section{Abstract}

Background-Freezing of gait (FOG) is a common symptom in patients with advanced Parkinson's disease (PD) representing a major cause of disability and falls. Although the pathophysiology of FOG remains poorly understood, non-dopaminergic pathways have been implicated. Treatment studies of levodopa and selegiline have shown limited benefit for FOG. Limited data suggest that amantadine, an N-methyl-D-aspartate receptor antagonist, may be beneficial for FOG in PD.

Objective-To examine the relationship between treatment with oral amantadine and FOG in patients with PD.

Methods-We conducted a retrospective chart review of PD patients who received amantadine specifically for FOG and had a follow-up assessment of FOG. The primary outcome measure was self-reported effectiveness of amantadine (improvement, worsening, or no change in FOG) based on records from the follow-up assessment.

Results-Eleven PD patients with median age of PD onset 67 (range 51-84) years and median Hoehn and Yahr stage 3 (range 2-4) met the study population selection criteria. Ten of 11 patients reported improvement in FOG after initiation of amantadine; FOG worsened in one patient. Median amantadine dose was $100 \mathrm{mg}$ twice daily and treatment duration 20 months (range 6-66). Four patients reported reduction in benefit after four months. Three patients reported adverse effects, including blurred vision, visual hallucinations and peripheral edema; the latter two effects resulted in discontinuation of amantadine.

Conclusion-Amantadine is associated with self-reported improvement in FOG in PD but this effect may be transient. Further studies, including a randomized placebo-controlled trial are needed to better evaluate this association.

\section{Keywords}

Amantadine; Freezing of Gait; Parkinson's disease

\footnotetext{
*Corresponding Author: Roneil Malkani, MD, MS, 710 N. Lake Shore Drive \#516, Chicago, IL 60611, Tel: (312) 503-1264, Fax: (312) 908-5073,r-malkani@fsm.northwestern.edu.

Conflicts of Interest: For the remaining authors, none were declared.

Financial disclosures:

Roneil Malkani has received research support from the American Sleep Medicine Foundation, honorarium from Allergan and American Physician Institute. Cindy Zadikoff has received honorarium for speakers bureau/consulting from Allergan, Merz, GSK, Teva, Ipsen, and Abbott. Tanya Simuni received research support from NINDS, MJFF, NPF, Northwestern Dixon Foundation, participates as a site PI in clinical trials sponsored by PSG, IMPAX, Synovia, TEVA, Phytopharm, served on the advisory boards and speaker for TEVA, GE, Novartis, Ibsen and received honoraria for consulting from Merz, Ibsen, GE, IMPAX, and Allergan. For the remaining authors, none were declared.
} 


\section{INTRODUCTION}

Freezing of gait (FOG) occurs in 53\% of patients with advanced Parkinson's disease (PD). This symptom is a major source of disability and is associated with increased risk of falls and injuries in the PD population. ${ }^{1}$ The pathophysiology of FOG is poorly understood but is thought to be driven by both dopaminergic and non-dopaminergic pathways. ${ }^{2}$ Although levodopa can improve FOG in patients with PD, improvement in FOG correlates poorly with improvement in rigidity, bradykinesia, and postural instability. ${ }^{2}$

With progression of PD, patients develop fluctuations in motor symptoms, which are partly related to the timing of medication administration. With the administration of medications, symptoms improve temporarily ("on" state). When the medication wears off, symptoms worsen ("off" state) until another dose of medication is taken. FOG is most commonly observed in the medication "off" state; "off" FOG can respond to dopaminergic therapy, particularly in early stages of PD. However, medication-resistant FOG that persists in the "on" state despite improvement of other parkinsonian symptoms is more frequent in advanced stages of PD and is challenging to treat.

There are limited treatment options for gait dysfunction, specifically FOG. Various medications have been tried to treat FOG but have shown limited benefit. In patients with PD and motor fluctuations, levodopa improves "off" FOG but may occasionally result in "on" FOG. ${ }^{2}$ In one study, selegiline, a monoamine oxidase-B inhibitor, was associated with mild improvement in FOG and reduced the risk of developing FOG, but other studies have yet to confirm this finding. ${ }^{3}$ A home physical therapy program using rhythmical cueing on gait and gait-related activity was associated with a small but transient benefit in freezing severity, gait speed, step length, and timed balance; however, this effect attenuated over 6 weeks if cueing was not continued. ${ }^{4}$

Amantadine is approved as adjunctive treatment for PD. The mechanism of action of amantadine is unclear but is thought to involve antagonism of $\mathrm{N}$-methyl D-aspartate (NMDA) receptors and inhibition of dopamine re-uptake. ${ }^{5,6}$ While some data suggest that amantadine is associated with lower risk of FOG, results are conflicting. $1,7,8$

The objective of this study is to examine the relationship between orally administrated amantadine and change in FOG among PD patients taking amantadine specifically for FOG. We hypothesized that treatment with oral amantadine is associated with improvement in FOG in PD patients.

\section{MATERIALS AND METHODS}

We conducted a retrospective chart review of PD patients treated with amantadine for FOG associated with PD at Northwestern University PD and Movement Disorders Center between $1 / 2 / 2004$ and $2 / 25 / 2009$. The protocol was approved by the Northwestern University Institutional Review Board. Eligible patients had to have at least one follow up visit after initiation of amantadine to assess FOG. Patients with records lacking documentation of treatment response were not eligible for study participation. Based on documentation, we were not able to distinguish whether FOG was in "on" and/or "off" states.

The primary outcome measure was subjective self-report about FOG during a follow up visit after initiating amantadine, classified as improved, worsened, or static FOG. Additional outcomes included duration of the clinical effect and adverse effects associated with amantadine use. Patient demographics and disease characteristics recorded included age, 
gender, age of PD onset, PD duration, Hoehn and Yahr stage (H\&Y), levodopa equivalent daily dose (LEDD), amantadine dose and frequency, and duration of treatment. ${ }^{9}$

Descriptive statistics including frequencies, means, and standard deviations were performed for outcome measures and patient characteristics using SPSS version 16 (SPSS Inc., Chicago, IL).

\section{RESULTS}

Two hundred thirty-six patients were treated with amantadine throughout the study period. Of this group, 196 had a diagnosis of PD, and 171 were given amantadine at the Northwestern University PD and Movement Disorders Center. Twelve patients were given amantadine specifically for FOG; 11 of these patients had a follow-up visit. The remaining 159 patients were excluded because they were given amantadine for another indication (tremor: 72; dyskinesia: 49; fatigue: 3 ; rigidity: 2 patients) or the indication was unclear (33 patients). Patient characteristics, treatment duration, amantadine dose, and response are presented in Table 1. The median age of PD onset and PD duration were 67 years (range 5184 years) and 4 years (1-11 years), respectively. Median H\&Y stage was 3 (range 2-4). One of the 11 patients was female. Median LEDD was $475 \mathrm{mg}$ (range 0-1348 mg). Median amantadine dose was $100 \mathrm{mg}$ twice daily (range $100 \mathrm{mg}$ once daily to $100 \mathrm{mg}$ twice daily), and median duration of therapy was 20 months (range 6-66 months).

Ten out of 11 patients reported subjective improvement in FOG after initiating amantadine. Treatment with amantadine was associated with worsening of FOG in one patient (\#2). In another patient (\#10), FOG initially improved but the benefit plateaued; the amantadine was therefore discontinued, but the patient soon developed worsening of FOG, so amantadine was restarted and the patient reported subjective improvement of gait. Four patients reported attenuation of benefit within 4 months. Two patients reported adverse effects resulting in discontinuation of amantadine. Patient \#8 developed lower extremity edema within 5 months which resolved when amantadine was discontinued. Patient \#11 developed hallucinations in the first 2 months of treatment which persisted despite reduction in amantadine dose from $100 \mathrm{mg}$ twice daily to $100 \mathrm{mg}$ once daily. Amantadine was therefore discontinued; however, this patient was also taking ropinirole and carbidopa/levodopa which may have contributed to the hallucinations. Patient \#7 reported blurred vision and temporarily stopped taking amantadine but resumed it after one month.

\section{DISCUSSION}

In our cohort, 10 of the 11 patients had subjective improvement in FOG after initiating treatment with oral amantadine. Four patients noted reduction in benefit after about 4 months. Adverse effects included visual hallucinations and peripheral leg edema, both which have been reported. ${ }^{8}$

Conflicting available data suggest that amantadine may be beneficial for FOG. A retrospective study demonstrated an association between longer duration of treatment with amantadine and decreased appearance of FOG; however, this association was seen in the univariate but not multivariate analysis. ${ }^{1}$ A recent investigation showed subjective improvement in FOG as assessed by the FOG-questionnaire with intravenous amantadine at dose of $200 \mathrm{mg}$ twice daily for two days. ${ }^{10}$ Improvement was seen in patients with FOG refractory to dopaminergic therapy, further supporting the concept that FOG may involve non-dopaminergic mechanisms. However, underlying clinical diagnoses in that study were heterogeneous (PD, multiple system atrophy, progressive supranuclear palsy, and primary freezing of gait), and responders had a lower age of disease onset. ${ }^{10}$ In contrast, a case 
control study of 6620 patients with PD suggested that amantadine may be associated with the presence of FOG. ${ }^{7}$ The results of our study suggest that oral amantadine may be beneficial for FOG associated with PD. While other studies examined the effect of amantadine on the development of FOG in patients with PD, our study and the study of intravenous amantadine have examined effects on existing FOG.

Although the mechanism of action of amantadine in PD is unclear, it may affect multiple pathways. In our study, response in FOG to amantadine was seen across the range of disease severity and large range of LEDD, suggesting that the mechanism of benefit in FOG from amantadine may include dopaminergic and non-dopaminergic mechanisms. Amantadine has been shown to stabilize the NMDA receptor channel in the closed conformation, thus antagonizing this receptor. ${ }^{5}$ Amantadine also increases extracellular dopamine levels, an effect which may be due to NMDA receptor inhibition. ${ }^{6}$ Additionally, studies in rat pheochromocytoma suggest that amantadine may stimulate gene expression of L-amino acid decarboxylase, the enzyme responsible for converting levodopa to dopamine. ${ }^{11}$

We recognize several limitations of this study. First, the sample size was small. Second, the data collection was retrospective and assessments were subjective and qualitative. Third, this study was not controlled; at least some of the improvement may be due to placebo effects. Fourth, the clinicians used a relatively small dose of amantadine so dose response effect was not explored. Finally, "off" and "on" freezing was not distinguished. Despite these limitations, this study provides the pilot data for further evaluation of the relationship between amantadine and FOG.

In summary, the results of this study indicate that amantadine is associated with selfreported improvement in FOG in patients with PD. The benefit, however, may be transient. Amantadine was well tolerated with occasional adverse effects at the dose up to $200 \mathrm{mg}$ daily. A randomized controlled trial examining the relationship between FOG and amantadine using objective measures is warranted and is underway.

\section{Acknowledgments}

Source of Funding: Roneil Malkani has received research support from the American Sleep Medicine Foundation, honorarium from Allergan and American Physician Institute. Cindy Zadikoff has received honorarium for speakers bureau/consulting from Allergan, Merz, GSK, Teva, Ipsen, and Abbott. Tanya Simuni received research support from NINDS, MJFF, NPF, Northwestern Dixon Foundation, participates as a site PI in clinical trials sponsored by PSG, IMPAX, Synovia, TEVA, Phytopharm, served on the advisory boards and speaker for TEVA, GE, Novartis, Ibsen and received honoraria for consulting from Merz, Ibsen, GE, IMPAX, and Allergan.

\section{References}

1. Giladi N, Treves TA, Simon ES, et al. Freezing of gait in patients with advanced Parkinson's disease. J Neural Transm. 2001; 108(1):53-61. [PubMed: 11261746]

2. Bartels AL, Balash Y, Gurevich T, et al. Relationship between freezing of gait (FOG) and other features of Parkinson's: FOG is not correlated with bradykinesia. J Clin Neurosci. 2003 Sep; 10(5): 584-8. [PubMed: 12948464]

3. Shoulson I, Oakes D, Fahn S, et al. Impact of sustained deprenyl (selegiline) in levodopa-treated Parkinson's disease: a randomized placebo-controlled extension of the deprenyl and tocopherol antioxidative therapy of parkinsonism trial. Ann Neurol. 2002 May; 51(5):604-12. [PubMed: 12112107]

4. Nieuwboer A, Kwakkel G, Rochester L, et al. Cueing training in the home improves gait-related mobility in Parkinson's disease: the RESCUE trial. J Neurol Neurosurg Psychiatry. 2007 Feb; 78(2):134-40. [PubMed: 17229744] 
5. Blanpied TA, Clarke RJ, Johnson JW. Amantadine inhibits NMDA receptors by accelerating channel closure during channel block. J Neurosci. 2005 Mar 30; 25(13):3312-22. [PubMed: 15800186]

6. Mizoguchi K, Yokoo H, Yoshida M, et al. Amantadine increases the extracellular dopamine levels in the striatum by re-uptake inhibition and by N-methyl-D-aspartate antagonism. Brain Res. 1994 Oct 31; 662(1-2):255-8. [PubMed: 7859080]

7. Macht M, Kaussner Y, Moller JC, et al. Predictors of freezing in Parkinson's disease: a survey of 6,620 patients. Mov Disord. 2007 May 15; 22(7):953-6. [PubMed: 17377927]

8. Singer C, Uzcategui G, Lyons K, et al. Efficacy and tolerability of amantadine: a retrospective review of old and new indications at a tertiary referral center. Mov Disord. 2002; 17(S5):S97.

9. Tomlinson CL, Stowe R, Patel S, et al. Systematic review of levodopa dose equivalency reporting in Parkinson's disease. Mov Disord. Research Support, Non-US Gov't Review. 2010 Nov 15; 25(15): 2649-53.

10. Kim YE, Yun JY, Jeon BS. Effect of intravenous amantadine on dopaminergic-drug-resistant freezing of gait. Parkinsonism Relat Disord. 2011 Jul; 17(6):491-2. [PubMed: 21463965]

11. Bennett VL, Juorio AV, Li XM. Possible new mechanism for the antiparkinsonian effect of amantadine. J Psychiatry Neurosci. 1999 Jan; 24(1):52-3. [PubMed: 9987209] 


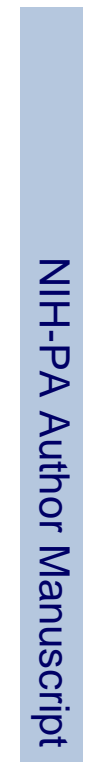

Malkani et al.

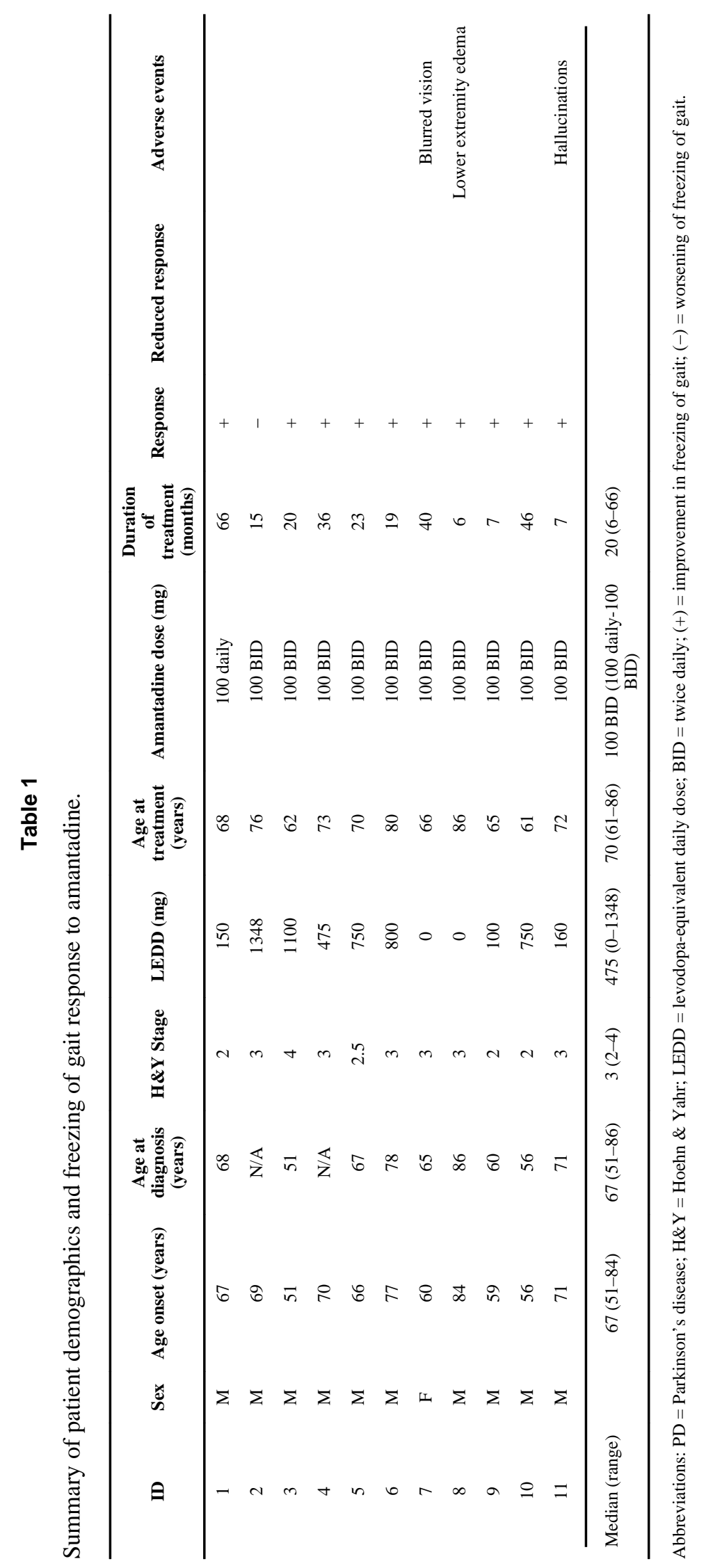

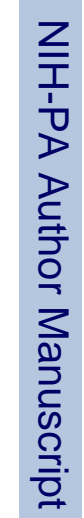

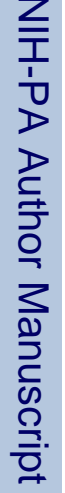

Clin Neuropharmacol. Author manuscript; available in PMC 2013 November 01. 